\title{
Evaluation of total phenolic content, antioxidant activity, germination power, and yield of pigeon pea (Cajanus cajan) sprouts elicited using various Na- alginate levels with different elicitation duration
}

\author{
1,2,* Ariviani, S., ${ }^{1}$ Hapsari, K.C., ${ }^{1}$ Fauza, G., ${ }^{1}$ Ishartani, D., ${ }^{1}$ Atmaka, W., ${ }^{1,2}$ Khasanah, L.U. \\ and ${ }^{1}$ Siswanti
}
${ }^{1}$ Department of Food Science and Technology, Faculty of Agriculture, Universitas Sebelas Maret, Jl. Ir. Sutami 36 A, Kentingan, Surakarta 57126, Indonesia
${ }^{2}$ Food, Nutrition, and Public Health Research Center, Universitas Sebelas Maret, Jl. Ir. Sutami 36 A, Kentingan, Surakarta 57126, Indonesia

\section{Article history:}

Received: 7 December 2020

Received in revised form: 16

January 2021

Accepted: 24 June 2021

Available Online: 4 July 2021

Keywords:

Antioxidant,

Germination power,

Yield,

Na-alginate,

Elicitation,

Pigeon pea

DOI:

https://doi.org/10.26656/fr.2017.5(S2).005

\begin{abstract}
This study was aimed to investigate the total phenolic content and antioxidant activity of elicited pigeon pea (Cajanus cajan) sprouts prepared using various Na-alginate concentrations at different elicitation durations. The germination power and yield of the sprouts were also examined. Elicitation proved capable to improve the antioxidant capacity of legumes sprouts but the yield and germination rates were decreased due to the elicitation stress. In this study, elicitation conducted by immersing pigeon pea seeds in $\mathrm{Na}$ -alginate solutions $(250,300$, and $350 \mathrm{ppm})$ for various duration $(16,20$, and $24 \mathrm{hrs})$. The seeds were then germinated for $48 \mathrm{hrs}$ to produce Na-alginate elicited pigeon pea sprouts. The results showed that the total phenolic content (TPC) and antioxidant activity (Trolox equivalent antioxidant capacity, TEAC, and Ferric reducing antioxidant power, FRAP) of elicited pigeon pea sprouts were significantly increased along with the increasing elicitation duration in all Na-alginate levels. The higher Na-alginate levels produce a higher level of TPC, TEAC, and FRAP values. On the other hand, germination power and the yield of the sprouts were significantly decreased along with increasing Na-alginate levels and elicitation duration. Elicitation using $350 \mathrm{ppm}$ Na-alginate with an elicitation duration of $24 \mathrm{hrs}$ produces elicited pigeon pea sprouts with the highest TPC and antioxidant activity, but lowest germination power and yield. These results have an important consequence in developing an elicitation technique to improve the antioxidant capacity of leguminous.
\end{abstract}

\section{Introduction}

Pigeon pea seed is proved as a potential source of bioactive molecules that promising to treat multiple diseases (Mathew et al., 2017). The health benefits of pigeon pea are mainly contributed by the phenolic compounds which are exhibited versatile bioactivities such as antitumor, antiviral, anti-inflammatory, antioxidant, hypocholesterolemic, and hypoglycemic activities (Gai et al., 2021). Ariviani et al. (2018) reported that pigeon pea seeds have the potential to be developed as an anti-diabetic functional drink related to their antioxidant capacity. It had been reported that the total phenolic content and radical scavenging activity of pigeon pea were higher than those of cowpea.

Germination is a common strategy to promote antioxidant compounds production as well as antioxidant activity of several legumes (Rajendra et al., 2019; Ariviani, Mudalifah, Ishartani et al., 2020). Several studies had reported improvement of antioxidant capacities such as total phenolic content (TPC) and radical scavenging activity (RSA) as well as the reducing power of pigeon pea through germination, which reported by Uchegbu and Ishiwu (2016), Uchegbu et al. (2017), and Sharma and Singh (2018).

Liu et al. (2019) stated that elicitation is a promising strategy rather than other conventional biotechnological techniques for increasing the bioactive compounds and biological activity of sprouts. Elicitors most widely used in the elicitation process for enhancing the accumulation of bioactive compounds in sprouts, among others are methyl jasmonate, salicylic acid, chitosan, sucrose, methionine, glutamic acid, $\mathrm{NaCl}$, mannitol, and light. Elicitation proved capable to increase the antioxidant 
capacity of legumes sprouts. The UV elicitation increases TPC and RSA of lentil sprouts (Swieca et al., 2014). Elicitation using mannitol effectively enhance the TPC, RSA and reducing power of lentil sprouts depend on mannitol concentration (Swieca, 2015a). $\mathrm{NaCl}$ elicitation improves total flavonoid content, RSA and reducing power of cowpea sprouts (Rajendra et al., 2019) and pigeon pea sprouts (Kristiani et al., 2019) depend on $\mathrm{NaCl}$ concentration and the elicitation duration, respectively. Swieca (2015b) stated that elicitation trigger desirable secondary metabolites production, include antioxidant compounds, but the elicitor concentration and elicitation duration required for optimum secondary metabolite accumulation are a characteristic of each plant species. Therefore, Investigation of antioxidant capacities such as TPC and antioxidant activities of legumes sprouts elicited using various elicitor levels and elicitation duration is crucial.

Elicitation effectively increased antioxidant capacity and had a significant impact on the sprouting percentage of germination rate of common bean (Phaseolus vulgaris) sprouts (Ampofo et al., 2020). Different elicitors used in the elicitation process produced different impacts on the antioxidant capacity and germination rate of kidney beans sprouts (Limón et al., 2014), and lentil sprouts (Peñas et al., 2015). Increasing elicitor concentration was necessary for maximizing the TPC of chickpea sprouts but it significantly reduces the germination power (León-López et al., 2020). The author's previous study showed that the antioxidant capacity of cowpea sprouts increased along with increasing $\mathrm{NaCl}$ concentration used as an abiotic elicitor (Rajendra et al., 2019). Other studies such as Swieca (2015a) and Saha et al. (2010) reported improvement of the antioxidant capacity of lentil and mung bean sprouts elicited with $\mathrm{NaCl}$ along with increasing $\mathrm{NaCl}$ levels. The antioxidant capacity of pigeon pea sprouts increases along with increasing $\mathrm{NaCl}$ elicitation duration (Kristiani et al., 2019). To the best of our knowledge, investigation on TPC and antioxidant activity, as well as germination power and yield of pigeon pea sprouts elicited using Naalginate with various concentrations and elicitation durations have not been reported yet. The study aims to evaluate the TPC, antioxidant activity which includes RSA (Trolox Equivalent Antioxidant Capacity, TEAC) dan Ferric Reducing Antioxidant Power (FRAP) (Ascorbic Acid Equivalent Activity, AAEA), germination power and yield of pigeon pea sprouts elicited using Na-alginate $(250,300,350 \mathrm{ppm})$ as biotic elicitor for different elicitation durations (16, 20, $24 \mathrm{hrs})$.

\section{Materials and methods}

The materials used in this study were pigeon pea obtained from Gunung Kidul, Yogyakarta, Indonesia; folin-ciocalteau's phenol reagent, ABTS (2,2'Azino-bis (3-ethylbenzothiazoline-6-sulfonic acid) diammonium salt), potassium persulfate, Trolox (6-Hydroxy-2, 5, 7, 8tetramethylchromane-2-carboxylic acid), ascorbic acid were obtained from Sigma-Aldrich Co. (St.Louis, MO, USA). Na-alginate, sodium carbonate, sodium dihydrogen phosphate dihydrate, disodium phosphate dihydrate, potassium ferricyanide, hydrochloric acid, Iron (III) chloride hexahydrate, trichloroacetic acid (TCA), Ethanol, and Methanol were purchased from Merck Millipore Co. (Darmstadt, Germany). All reagents used in this study were analytical grade.

\subsection{Pigeon pea sprouts preparation}

Pigeon pea sprouts were prepared as previously described (Kristiani et al., 2019; Rajendra et al., 2019; Ariviani, Mudalifah, Ishartani et al., 2020) with slight modification. Briefly, the sorted pigeon pea seeds were rinsed three times using distilled water. Furthermore, the seeds were exposed to elicitation by submerging in Naalginate solution $(250,300$, and $350 \mathrm{ppm})$ with a ratio of $1: 3 \mathrm{w} / \mathrm{v}$ for various elicitation durations $(16,20,24 \mathrm{hrs})$, then germinated for $48 \mathrm{hrs}$.

\subsection{Preparation of pigeon pea sprouts extract}

The pigeon pea sprouts extract preparation was conducted according to Ariviani, Lainuna, and Fauza (2020) with slight modification. Briefly, the dehulled and mashed pigeon pea sprouts $(2 \mathrm{~g})$ were extracted with 20 $\mathrm{mL}$ of methanol $(80 \%)$ using a water bath shaker (SWB 20, Fisher Scientific Haake, Germany) at $200 \mathrm{rpm}, 50^{\circ} \mathrm{C}$ for 2 hours. The methanol extract was separated using PLC-05 Centrifuge (Gemmy, Taiwan) at $10000 \mathrm{rpm}$ for 15 minutes. The clear supernatant was collected in dark bottles and stored at $10^{\circ} \mathrm{C}$ until further analysis.

\subsection{Total phenolic content determination}

The total phenolic content of pigeon pea sprout extracts was analyzed using Follin-Ciocalteu Reagent as described by Singleton et al. (1999). The total phenolic content was expressed as Gallic Acid Equivalent $(\mu \mathrm{M}$ GAE/g sample dry weight).

\subsection{Antioxidant activity analysis}

Antioxidant activity analysis conducted by measuring the radical scavenging activity (RSA) and Ferric reducing antioxidant power (FRAP). RSA determined using the ABTS $\bullet+$ free radical method $(\operatorname{Re}$ et al., 1999) and expressed as Trolox Equivalent 
Antioxidant Capacity (mM TEAC/g sample dry weight). FRAP measured using the method described by Berker et al. (2007) and expressed as Ascorbic Acid Equivalent Activity ( $\mu \mathrm{M}$ AAEA/g sample dry weight)

\subsection{Germination power and yield determination}

The germination power was determined by calculating the ratio of the number of pigeon pea seeds that sprouted to the total number of the seeds and expressed as a percentage (\%). The yield was determined based on the ratio of dry weight of pigeon pea sprouts to the initial pigeon pea seeds and expressed as a percentage $(\%)$

\subsection{Statistical analysis}

The Data was presented as a mean \pm standard deviation from three replication. IBM SPSS Statistics 22 (SPSS Inc., Chicago, USA) program with the General Linear Model (Univariate Analysis of Variance) was used for data analysis. Duncan's Multiple Range Test (DMRT) was used to evaluate the significant difference between means $(p<0.05)$ for each factor.

\section{Results and discussion}

\subsection{Total phenolic content (TPC)}

TPC of pigeon pea sprouts prepared with various $\mathrm{Na}$ -alginate concentrations and elicitation durations was presented in Figure 1A. The TPC values $(\mu \mathrm{M}$ GAE/g sample dry weight) of pigeon pea sprouts elicited using various Na-alginate levels with elicitation duration of 16 , 20 , and $24 \mathrm{hrs}$ were $9729.2 \pm 105.4,10611.4 \pm 70.6$, and $11374.2 \pm 97.8$ for $250 \mathrm{ppm}$ Na-alginate, $10328.1 \pm 95.8$, $11172.7 \pm 85.9$, and $12066.5 \pm 101.8$ for $300 \mathrm{ppm} \mathrm{Na}-$ alginate, $\quad 11864.5 \pm 63.1, \quad 12659.1 \pm 100.9, \quad$ and $13587.5 \pm 90.3$ for $350 \mathrm{ppm} \mathrm{Na-alginate.}$

Based on the statistical analysis, the TPC was affected by Na-alginate concentration and elicitation duration. Increasing of elicitation duration produce significantly higher TPC in all Na-alginate concentration. For each elicitation duration, a higher Naalginate concentration produces pigeon pea sprouts with significantly higher TPC. The highest TPC of pigeon pea sprouts produced by elicitation using $350 \mathrm{ppm} \mathrm{Na-}$ alginate for $24 \mathrm{hrs}$. This is possible because the higher either the Na-alginate concentration or the elicitation duration induced more plant stress levels. The higher stress levels stimulate higher secondary metabolite production, including enzymatic and non-enzymatic antioxidant production such as phenolic biosynthesis as the stress response (Swieca, 2015a; Swieca, 2015b; Swieca, 2016). Several studies reported a similar result, the total phenolic and total flavonoid content of elicited legumes sprouts increases along with increasing elicitor concentrations, such as in cowpea and lentil (Swieca, 2015a; Rajendra et al., 2019).

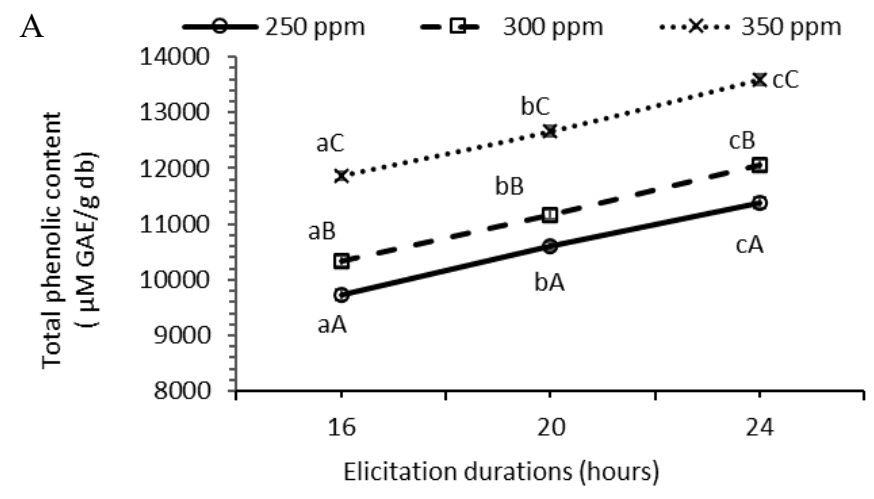

B
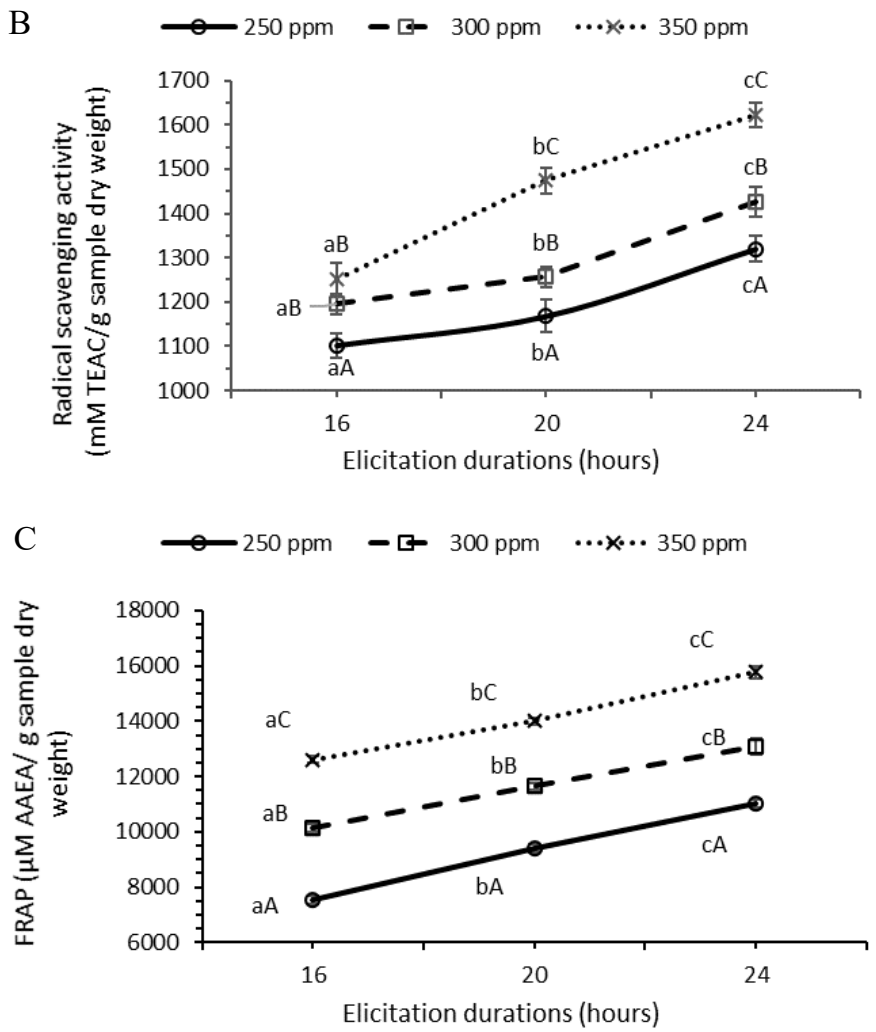

Figure 1. Total phenolic content (A), radical scavenging activity (B) and Ferric Reducing Antioxidant Power (FRAP) (C) of elicited pigeon pea sprouts prepared using various Naalginate concentration with different elicitation durations. Different capital letters mean significant differences $(p<0.05)$ for different Na-alginate concentration with same elicitation duration, whereas different lowercase letters mean significant differences for different elicitation duration with the same Naalginate concentration.

Na-alginate is one kind of carbohydrate biotic elicitor that capable to improve the production of plant secondary metabolites (Thakur et al., 2019). Carbohydrate is classified as "general elicitors" which induce non-specific mechanisms for the trigger of defense response in different plant cultures (Baenas et al., 2014). Elicitors act as plant stress inducer, which will promote the biosynthesis of specific compounds that have an important role in the plant's defense system to 
overcome the stress conditions (Ramirez-estrada et al., 2016). Specific elicitors such as salt $(\mathrm{NaCl})$ induce salinity stress which further triggers osmotic stress tolerance through proline formation as an osmolyte. Proline synthesis is related to overproduction of erythrose-4-phosphate that plays a role in the shikimate pathway which stimulate phenolic compounds synthesis (Shetty, 2004; Thakur and Sharma, 2005; Saha et al., 2010)

\subsection{Antioxidant activity}

The antioxidant activity of pigeon pea sprout extracts was determined by measuring the radical scavenging activity (TEAC) and the reducing power (FRAP). The results were presented in Figure $1 \mathrm{~B}$ and 1C. The TEAC values (mM TEAC/ $g$ sample dry weight) of pigeon pea sprouts elicited using various Na-alginate levels for 16 , 20 , and $24 \mathrm{hrs}$ elicitation durations were $1102.4 \pm 27.4$, $1168.5 \pm 37.3, \quad 1319.8 \pm 29.2 \quad(250 \mathrm{ppm} \quad$ Na-alginate $)$, $1195.9 \pm 22.9,1256.5 \pm 23.0,1426.5 \pm 34.3(300 \mathrm{ppm} \mathrm{Na}-$ alginate), and $1252.0 \pm 34.9,1474.4 \pm 29.5,1622.5 \pm 28.8$ (350 ppm Na-alginate), respectively. While the FRAP values ( $\mu \mathrm{M}$ AAEA/ $g$ sample dry weight) of the same sample were $\quad 7539.4 \pm 134.1, \quad 9406.3 \pm 153.5$, $11042.1 \pm 147.1$ (250 ppm Na-alginate), $10149.0 \pm 132.9$, $11641.5 \pm 127.8,13087.2 \pm 304.9$ (300 ppm Na-alginate), and $12612.8 \pm 133.6,14004.9 \pm 113.4,15778.1 \pm 216.4(350$ ppm Na-alginate).

Both the TEAC and FRAP of Na-alginate elicited in pigeon pea sprouts were increased along with increasing $\mathrm{Na}$-alginate concentration in all elicitation duration. It was also observed that the longer elicitation duration produce higher levels of the TEAC and FRAP in all Naalginate concentrations. The highest TEAC and FRAP of pigeon pea sprouts produced by elicitation using 350 ppm Na-alginate for $24 \mathrm{hrs}$. The improvements of the TEAC and FRAP observed were in line with the total phenolic content enhancement due to the increasing of Na-alginate levels as well as elicitation durations as presented in Figure 1A. Plant phenolic compounds can act as a radical scavenger by donating hydrogen atom or electrons and also as reducing agents (Craft et al., 2012; Gulcin, 2020). Several studies were reported the significant positive correlation between TPC and TEAC as well as TPC and FRAP of legumes extract. Lu et al. (2018) reported the significant correlation between TPC and TEAC $(\mathrm{r}=0.897, \mathrm{p}<0.01)$, TPC and FRAP (0.943, $\mathrm{p}<0.01$ ) of faba bean (Vicia faba L.) seed extract. The TPC of grass pea (Lathyrus sativus) seed extract was correlated to the TEAC $(\mathrm{r}=0.881)$ and the FRAP $(\mathrm{r}=$ 0.781) (Rybiński et al., 2018). There was significant correlation between TPC and TEAC $(\mathrm{r}=0.901-0.933$, $\mathrm{p}<0.05)$, TPC and FRAP $(0.786-0.877, \mathrm{p}<0.05)$ of nonoil seed legumes extracts (Diniyah et al., 2020). The results on TPC and antioxidant activity indicated that the best Na-alginate elicitation technique for improving the antioxidant capacity of pigeon pea sprouts through submerged in $350 \mathrm{ppm}$ Na-alginate for $24 \mathrm{hrs}$ before germination.

\subsection{Germination power and yield}

The germination power and yield of the pigeon pea sprouts presented in Figure 2A and $2 \mathrm{~B}$ indicated that both the germination power and yield of the sprouts decreased along with increasing Na-alginate concentrations and elicitation durations. The lowest germination power and yield of pigeon pea sprouts were observed in elicitation using $350 \mathrm{ppm} \mathrm{Na-alginate} \mathrm{for} 24$ hrs. The result possible due to more severity of the stress effect as the consequence of higher elicitor concentration and longer elicitation durations as mentioned in section 3.1. The stress due to biotic elicitation inhibits the legumes plant's growth and development and further impact the yield decreases (Rasool et al., 2015). The higher level of elicitor concentration significantly reduces the germination power of lentil, mung bean, and chickpea sprouts (Dutta and Bera, 2014; Ouji, 2015; León-López et al., 2020). The research results suggested that the optimization study was needed to develop the $\mathrm{Na}$ -alginate elicitation technique as an effective strategy for antioxidant capacity improvement of pigeon pea sprouts that has a minimally impact on the inhibition of germination rate and the yield reduction.
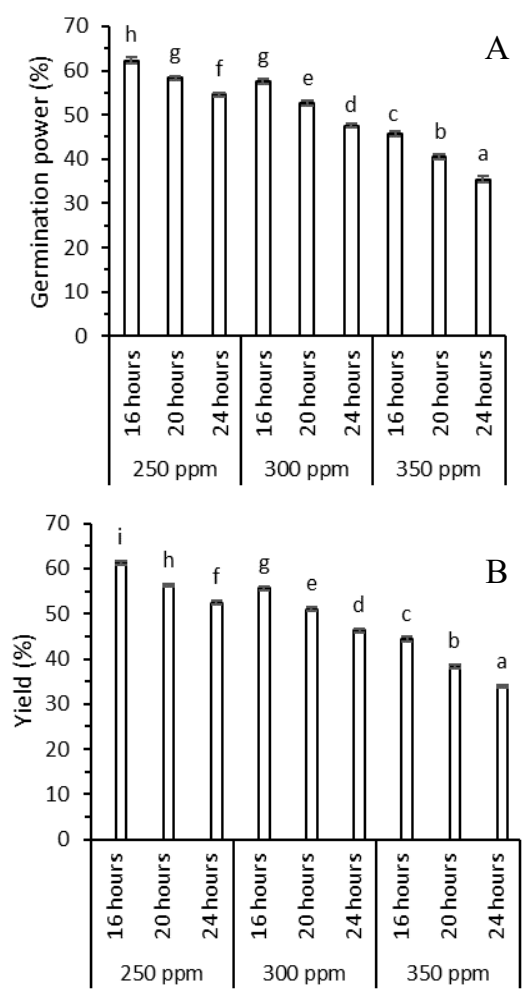

Figure 2. Germination power (A) and yield (B) of elicited pigeon pea sprouts prepared with different Na-alginate concentration and elicitation durations. Different letters mean significant differences $(p<0.05)$. 


\section{Conclusion}

Increased Na-alginate levels as well as the elicitation durations proved capable to significantly increase the TPC, RSA and FRAP but reduce the germination power and yield of Na-alginate elicited pigeon pea sprouts. Elicitation by submerging pigeon pea seeds in $350 \mathrm{ppm}$ $\mathrm{Na}$-alginate for $24 \mathrm{hrs}$ produces pigeon pea sprouts with the highest TPC, RSA and FRAP but the lowest germination power and yield. The research results have an important consequence in developing carbohydrate as general elicitors to increase antioxidant compounds as well as antioxidant activity of legumes sprouts.

\section{Conflict of interest}

The authors declare no conflict of interest.

\section{Acknowledgments}

This research was financially supported by National Competitive Research Grant for Fundamental Research, Ministry of Research and Technology Directorate of Higher Education 2019 with the contract number of 516/ UN27.21/ PN/ 2019.

\section{References}

Ampofo, J., Ngadi, M. and Ramaswamy, H.S. (2020). The impact of temperature treatments on elicitation of the phenylpropanoid pathway, phenolic accumulations and antioxidative capacities of common bean (Phaseolus vulgaris) sprouts. Food and Bioprocess Technology, 13(9), 1544-1555. https://doi.org/10.1007/s11947-020-02496-9

Ariviani, S., Affandi, D.R., Listyaningsih, E. and Handajani, S. (2018). The potential of pigeon pea (Cajanus cajan) beverage as an anti-diabetic functional drink. IOP Conference Series: Earth and Environmental Science, 102(1), 1-9. https:// doi.org/10.1088/1755-1315/102/1/012054

Ariviani, S., Lainuna, N. and Fauza, G. (2020). The potential of $\mathrm{NaCl}$ elicitation on improving antioxidant capacity and functional properties of sprouted pigeon pea (Cajanus cajan) flour. AIP Conference Proceedings, 2219(1), 070005. https:// doi.org/10.1063/5.0003642

Ariviani, S., Mudalifah, I., Ishartani, D. and Fauza, G. (2020). Investigation on antioxidant activity, protein, and whiteness degree of elicited cowpea sprouts flour prepared with various drying technique. AIP Conference Proceedings, 2219(1), 070003. https:// doi.org/10.1063/5.0003640

Baenas, N., García-Viguera, C. and Moreno, D.A. (2014). Elicitation: a tool for enriching the bioactive composition of foods. Molecules, 19, 13541-13563. https://doi.org/10.3390/molecules190913541

Berker, K.I., Güçlü, K., Tor, İ. and Apak, R. (2007). Comparative evaluation of $\mathrm{Fe}$ (III) reducing powerbased antioxidant capacity assays in the presence of phenanthroline, batho-phenanthroline, tripyridyltriazine (FRAP), and ferricyanide reagents. Talanta, 72(3), 1157-1165. https://doi.org/10.1016/ j.talanta.2007.01.019

Craft, B.D., Kerrihard, A.L., Amarowicz, R. and Pegg, R.B. (2012). Phenol-based antioxidants and the in vitro methods used for their assessment. Comprehensive Reviews in Food Science and Food Safety, 11(2), 148-173. https://doi.org/10.1111/ j.1541-4337.2011.00173.x

Diniyah, N., Badrul Alam, M. and Lee, S.H. (2020). Antioxidant potential of non-oil seed legumes of Indonesian's ethnobotanical extracts. Arabian Journal of Chemistry, 13(5), 5208-5217. https:// doi.org/10.1016/j.arabjc.2020.02.019

Dutta, P. and Bera, A.K. (2014). Effect of $\mathrm{NaCl}$ salinity on seed germination and seedling growth of mungbean cultivars. Legume Research - An International Journal, 37(2), 161-164. https:// doi.org/10.5958/j.0976-0571.37.2.024

Gai, Q., Jiao, J., Wang, X., Fu, Y., Lu, Y., Liu, J., Wang, Z.Y. and $\mathrm{Xu}, \mathrm{X}$.-J. (2021). Simultaneous quantification of eleven bioactive phenolic compounds in pigeon pea natural resources and in vitro cultures by ultra-high performance liquid chromatography coupled with triple quadrupole mass spectrometry (UPLC-QqQ-MS/MS). Food Chemistry, 335, 127602. https://doi.org/10.1016/ j.foodchem.2020.127602

Gulcin, İ. (2020). Antioxidants and antioxidant methods: an updated overview. In Archives of Toxicology. Archives of Toxicology, 94, 651-715. https:// doi.org/10.1007/s00204-020-02689-3

Kristiani, L.S., Rajendra, F.M. and Ariviani, S. (2019). Antioxidant capacity of pigeon pea (Cajanus cajan L.) sprouts elicited using $\mathrm{NaCl}$ with various elicitation time. IOP Conference Series: Materials Science and Engineering, 633, 012039. https:// doi.org/10.1088/1757-899X/633/1/012039

León-López, L., Escobar-Zúñiga, Y., Salazar-Salas, N.Y., Mora Rochín, S., Cuevas-Rodríguez, E.O., Reyes-Moreno, C. and Milán-Carrillo, J. (2020). Improving Polyphenolic Compounds: Antioxidant Activity in Chickpea Sprouts through Elicitation with Hydrogen Peroxide. Foods, 9(12), 1791. https:// doi.org/10.3390/foods9121791

Limón, R.I., Peñas, E., Martínez-Villaluenga, C. and 
Frias, J. (2014). LWT - Food Science and Technology Role of elicitation on the healthpromoting properties of kidney bean sprouts. $L W T$ Food Science and Technology, 56(2), 328-334. https://doi.org/10.1016/j.lwt.2013.12.014

Liu, H., Kang, Y., Zhao, X., Liu, Y., Zhang, X. and Zhang, S. (2019). Effects of elicitation on bioactive compounds and biological activities of sprouts. Journal of Functional Foods, 53(13), 136-145. https://doi.org/10.1016/j.jff.2018.12.019

Lu, Y.H., Tian, C.R., Gao, C.Y., Wang, B.N., Yang, W.Y., Kong, X., Chai, L.Q., Chen, G.C., Yin, X.F. and He, Y.H. (2018). Phenolic composition, antioxidant capacity and inhibitory effects on $\alpha$ glucosidase and lipase of immature faba bean seeds. International Journal of Food Properties, 21(1), 2366-2377. https:// doi.org/10.1080/10942912.2018.1522331

Mathew, D., Lidiya John, P., Manila, T.M. Divyasree, P. and Sandhya Rajan, V.T.K. (2017). Therapeutic molecules for multiple human diseases identified from pigeon pea (Cajanus cajan L. Millsp.) through GC-MS and molecular docking. Food Science and Human Wellness, 6(4), 202-216. https:// doi.org/10.1016/j.fshw.2017.09.003

Ouji, A. (2015). Effect of salinity stress on germination of five tunisian lentil (Lens culinaris L.) genotypes. European Scientific Journal, 11(21), 63-75.

Peñas, E., Limón, R.I., Martínez-Villaluenga, C., Restani, P., Pihlanto, A. and Frias, J. (2015). Impact of Elicitation on Antioxidant and Potential Antihypertensive Impact of Elicitation on Antioxidant and Potential Antihypertensive Properties of Lentil Sprouts. Plant Foods for Human Nutrition, 70, 401-407. https://doi.org/10.1007/ s11130-015-0508-3

Rajendra, F.M., Kristiani, L.S. and Ariviani, S. (2019). Elicitation under salinity stress increases flavonoid content and antioxidant activity in cowpea ( Vigna unguiculata ) sprouts. IOP Conference Series: Materials Science and Engineering, 633, 012034. https://doi.org/10.1088/1757-899X/633/1/012034

Ramirez-estrada, K., Vidal-limon, H., Hidalgo, D., Moyano, E., Golenioswki, M., Cusidó, R.M. and Palazon, J. (2016). Elicitation , an effective strategy for the biotechnological production of bioactive high -added value compounds in plant cell factories. Molecules, 21(182), 1-24. https://doi.org/10.3390/ molecules 21020182

Rasool, S., Latef, A.A.H.A. and Ahmad, P. (2015). Chickpea: Role and responses under abiotic and biotic stress. In Azooz, M.M. and Ahmad, P. (Eds.). Legumes under Environmental Stress: Yield,
Improvement and Adaptation. $1^{\text {st }}$ ed., p. 67-79. Oxford, UK: John Wiley and Sons, Ltd. https:// doi.org/10.1002/9781118917091.ch4

Re, R., Pellegrini, N., Proteggente, A., Pannala, A., Yang, M. and Rice-Evans, C. (1999). Antioxidant activity applying improved ABTS radical cation decolorization assay. Free Radical Biology and Medicine, 26(9-10), 1231-1237. https:// doi.org/10.1016/S0891-5849(98)00315-3

Rybiński, W., Karamać, M., Sulewska, K., Börner, A. and Amarowicz, R. (2018). Antioxidant potential of grass pea seeds from European countries. Foods, 7 (9), 142. https://doi.org/10.3390/foods 7090142

Saha, P., Chatterjee, P. and Biswas, A.K. (2010b). NaCl pretreatment alleviates salt stress by enhancement of antioxidant defense system and osmolyte accumulation in mungbean (Vigna radiata L . Wilczek ). Indian Journal of Experimental Biology, 48(6), 593-600.

Sharma, S. and Singh, A. (2018). Characterization of in vitro antioxidant activity, bioactive components, and nutrient digestibility in pigeon pea (Cajanus cajan) as influenced by germination time and temperature. Journal of Food Biochemistry, 43(2), 1-13. https:// doi.org/10.1111/jfbc.12706

Shetty, K. (2004). Role of proline-linked pentose phosphate pathway in biosynthesis of plant phenolics for functional food and environmental applications : a review. Process Biochemistry, 39(7), 789-803. https://doi.org/10.1016/S0032-9592(03)00088-8

Singleton, V.L., Orthofer, R. and Lamuela-Raventos, R.M. (1999). Analysis of total phenols and other oxidation substrates and antioxidants by means of folin-ciocalteu reagent. Methods in Enzymology, 299, 152-178. https://doi.org/10.1016/S0076-6879 (99)99017-1

Swieca, M. (2015a). Elicitation with abiotic stresses improves pro-health constituents, antioxidant potential and nutritional quality of lentil sprouts. Saudi Journal of Biological Sciences, 22(4), 409416. https://doi.org/10.1016/j.sjbs.2014.12.007

Swieca, M. (2015b). Production of ready-to-eat lentil sprouts with improved antioxidant capacity: Optimization of elicitation conditions with hydrogen peroxide. Food Chemistry, 180, 219-226. https:// doi.org/10.1016/j.foodchem.2015.02.031

Swieca, M. (2016). Potentially bioaccessible phenolics, antioxidant activity and nutritional quality of young buckwheat sprouts affected by elicitation and elicitation supported by phenylpropanoid pathway precursor feeding. Food Chemistry, 192, 625-632. https://doi.org/10.1016/j.foodchem.2015.07.058 
Swieca, M., Seczyk, L. and Gawlik-Dziki, U. (2014). Elicitation and precursor feeding as tools for the improvement of the phenolic content and antioxidant activity of lentil sprouts. Food Chemistry, 161, 288295. https://doi.org/10.1016/j.foodchem.2014.04.012

Thakur, M. and Sharma, A.D. (2005). Salt-stress-induced proline accumulation in germinating embryos: Evidence suggesting a role of proline in seed germination. Journal of Arid Environments, 62(3), 517-523. https://doi.org/10.1016/ j.jaridenv.2005.01.005

Thakur, M., Bhattacharya, S., Khosla, P.K. and Puri, S. (2019). Improving production of plant secondary metabolites through biotic and abiotic elicitation. Journal of Applied Research on Medicinal and Aromatic Plants, 12, 1-12. https://doi.org/10.1016/ j.jarmap.2018.11.004

Uchegbu, N.N. and Ishiwu, C.N. (2016). Germinated pigeon pea (Cajanus cajan): a novel diet for lowering oxidative stress and hyperglycemia. Food Science and Nutrition, 4(5), 772-777. https:// doi.org/10.1002/fsn3.343

Uchegbu, N.N., Okoli, E.C. and Onwurafor, E.U. (2017). Antioxidant properties of steamed paste (moin-moin) made from sprouted pigeon pea flour as influenced by heat treatment. American Journal of Food Technology, 13(1), 42-47. https://doi.org/10.3923/ ajft.2018.42.47 